\title{
UMA REVISÃO SOBRE O EFEITO TOMS - O FENÔMENO ONDE MACROMOLÉCULAS ATENUAM A TURBULÊNCIA EM UM LÍQUIDO
}

\author{
Vanessa C. Bizotto \\ Centro de Estudos de Petróleo, Universidade Estadual de Campinas, 13084-862 Campinas - SP, Brasil \\ Melissa I. Alkschbirs e Edvaldo Sabadini* \\ Instituto de Química, Universidade Estadual de Campinas, CP 6154, 13084-862 Campinas - SP, Brasil
}

Recebido em 10/5/10; aceito em 19/10/10; publicado na web em 18/2/11

\begin{abstract}
A REVISION ON THE TOMS EFFECT - A PHENOMENON IN WHICH MACROMOLECULES ATTENUATE TURBULENCE IN LIQUIDS. It is very well known that the addition of polymers to a liquid increases the shear viscosity of the solution. In other words, the polymer increases the dissipation of the flow energy. Contrarily, in turbulent flow, some particular macromolecules in very low concentration are able to produce large attenuation in the turbulence and thus, decreasing the dissipation of the energy. This article present a brief revision about macroscopic and molecular models used to explain this dynamic effect. Some of the experimental techniques used to quantify the attenuation of the turbulence and the main active substances are also discussed.
\end{abstract}

Keywords: drag reduction; polymer solutions; wormlike micelles.

\section{HISTÓRICO}

Os primeiros relatos sobre a redução de atrito hidrodinâmico (RAH) datam de 1906, descrevendo o comportamento anômalo do escoamento de pasta de papel. ${ }^{1}$ Entretanto, em 1883, em testes para calibrar bombas, já se observava a ocorrência de flutuações de cerca de $10 \%$ na vazão de água de reservatórios através de tubos. Muitos anos mais tarde, estas variações foram atribuídas à presença de substâncias de natureza polimérica produzidas por algas. ${ }^{2}$

Apesar do fenômeno da redução de atrito hidrodinâmico (RAH) não ser muito conhecido entre os químicos, sua descoberta (ou pelo menos sua demonstração sistemática) foi feita pelo químico britânico B. A. Toms, em 1948. Toms mostrou que uma solução diluída de poli(metil metacrilato) em monoclorobenzeno, sob certas condições de escoamento turbulento, oferecia menor resistência ao escoamento quando comparada ao solvente puro. $\mathrm{O}$ fenômeno passou então a ser conhecido como Efeito Toms, apesar da existência de outros termos como Efeito Texas (devido aos trabalhos sobre RAH desenvolvidos por pesquisadores do estado do Texas). ${ }^{3}$ Em 1960, foi observado que a goma guar era capaz de produzir RAH em soluções aquosas. ${ }^{4}$ Surgiu então a primeira aplicação do fenômeno citada nas refs. $1 \mathrm{e}$ 3: o uso de gomas em suspensões de areia em água para melhorar a fratura de poços de petróleo. Nos anos seguintes, um grande número de artigos e comunicações sobre RAH foi publicado em periódicos das mais diversas áreas do conhecimento, como matemática, física e engenharias, como indicado nas refs. 1 e 3 . A multidisciplinaridade de áreas pode ser entendida, uma vez que o fenômeno envolve conceitos de turbulência e dinâmica de polímeros em solução. ${ }^{3}$

\section{O FENÔMENO}

Uma das mais emblemáticas evidências do efeito de RAH está mostrada na Figura 1, na qual os bombeiros de Nova York demonstram a potencialidade do Efeito Toms para lançar água em maiores

\footnotetext{
*e-mail: sabadini@iqm.unicamp.br
}

distâncias. ${ }^{5}$ No ensaio, a mesma bomba impulsiona, através de duas mangueiras de mesmo comprimento e diâmetro, apenas água em uma delas, e água contendo 30 ppm de poli(óxido de etileno) em outra.

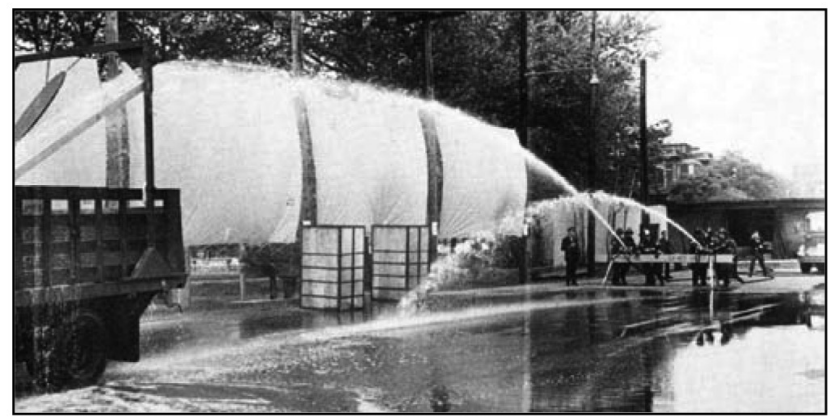

Figura 1. Ensaio realizado pelos bombeiros de Nova York, no final da década de 60, para demonstrar o potencial do efeito de redução de atrito hidrodinâmico. Uma bomba impulsiona água por uma das mangueiras e água contendo 30 ppm de poli(óxido de etileno) por outra. Reproduzida da ref. 5 , com permissão da Elsevier

Do ponto de vista físico-químico, esta imagem pode motivar reflexões sobre a razão para tamanha diferença de comportamento, causada pela ínfima quantidade do polímero dissolvido na água. Podese antecipar que "praticamente tudo" sobre o fenômeno ainda está para ser entendido. Como justificativa para esta afirmação, pode-se pensar na pequena estória apócrifa sobre o grande físico Werner Heisenberg, que teria dito que gostaria de fazer duas perguntas para Deus: 1- por que razão a relatividade é tão estranha? e 2- como explicar a turbulência? Ele concluía dizendo que Deus saberia a resposta da primeira questão. ${ }^{6}$ A natureza da turbulência é ainda um dos grandes desafios da física.

A combinação de turbulência e do caráter altamente diluído das soluções poliméricas torna o tratamento do fenômeno bastante complicado. As teorias moleculares são ainda primitivas, apesar de que, do ponto de vista hidrodinâmico, a RAH está relativamente 
bem estabelecida. $\mathrm{O}$ efeito altera a estabilidade da região laminar e a transição para turbulência. ${ }^{7}$ A região laminar caracteriza-se pelo deslizamento em baixas velocidades de camadas paralelas durante $o$ escoamento do fluido. Aumentando-se gradativamente a velocidade de escoamento, surgem estruturas denominadas de vórtices, sendo que isto ocorre quando as forças inerciais superam as forças viscosas no fluido. Estas estruturas, em certas condições, dissipam energia e tornam o escoamento caótico, caracterizando o regime turbulento. ${ }^{8}$

O parâmetro que caracteriza o regime de escoamento é o Número de Reynolds (Re), definido a partir da viscosidade ( $\eta$ ), da densidade do fluido $(\rho)$, da velocidade de escoamento $(v)$ e também das geometrias nas quais os ensaios são desenvolvidos. ${ }^{8}$ Para experimentos feitos em tubos capilares de diâmetro D, o Re é definido como:

$$
\operatorname{Re}=\frac{\rho v D}{\eta}
$$

Em tubos, a turbulência é atingida para valores de Re maiores do que cerca de $2300 .{ }^{8}$ A partir deste valor, as substâncias redutoras de atrito começam a atuar, conforme apresentado na Figura 2. O diagrama esquemático indica o comportamento hidrodinâmico idealizado de um determinado fluido puro (como, por exemplo, a água) e, comparativamente, os efeitos da presença do redutor de atrito hidrodinâmico. Ao entrar na região turbulenta, para o solvente puro (linha contínua) a vazão não se mantém proporcional, na medida em que Re aumenta. A linha tracejada representa a vazão fictícia que seria obtida, caso o fluido não perdesse energia pela turbulência. De certa forma, o agente redutor de atrito (representado no gráfico pelos pontos $\mathrm{x}$ ) consegue "prolongar" a região laminar, uma vez que é capaz de atenuar as estruturas dissipativas mantendo, desta forma, a vazão proporcionalmente alta. Esta é a assinatura do efeito de redução do atrito hidrodinâmico, e a porcentagem de redução de atrito hidrodinâmico que seria obtida para determinado Re poderia ser estimada pela diferença relativa das vazões.

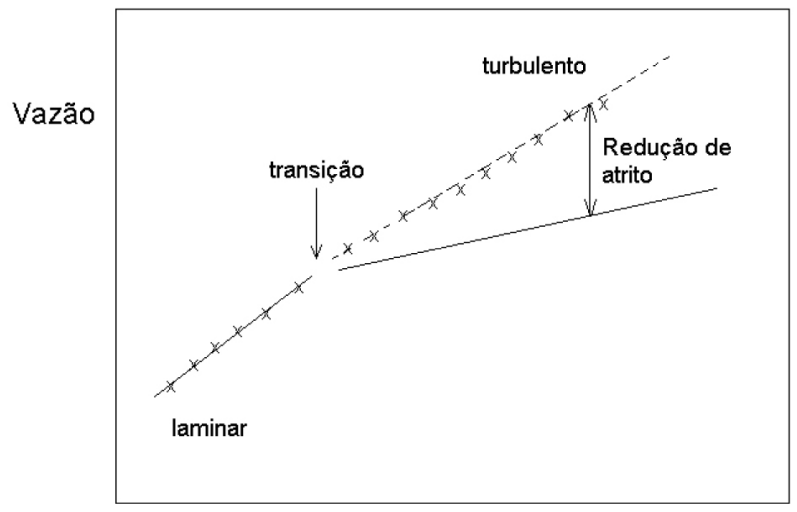

Número de Reynolds

Figura 2. Diagrama característico representando a variação da vazão de um líquido puro (linha contínua) e contendo um aditivo redutor de atrito (x), em função do número de Reynolds. As regiões laminar e turbulenta (região na qual o aditivo atua) estão representadas

\section{A redução de atrito hidrodinâmico do ponto de vista molecular}

Fundamentalmente, os modelos existentes (que são qualitativos) sugerem que as moléculas que agem como redutoras de atrito interferem na produção, no desenvolvimento ou no transporte da turbulência. Os principais modelos podem ser agrupados por abordarem aspectos relacionados com: dimensão, escala de tempo e de energia. ${ }^{9}$

$\mathrm{O}$ modelo de Virk $^{10}$ (relacionado com aspectos dimensionais) procura correlacionar o início da redução de atrito (no diagrama da
Figura 2, o ponto onde se inicia a diferenciação do solvente puro em relação à solução) com a razão entre o tamanho do polímero em solução (representado pelo seu raio de giração) e a dimensão da turbulência (" tamanho do vórtice", que será discutido adiante).

Os modelos que consideram a escala temporal prevêem que o início da redução de atrito ocorre quando as frequências para relaxação do polímero (relacionada com o processo de estiramento e contração da cadeia) e as associadas com o aparecimento dos vórtices são próximas. Segundo Lumley, ${ }^{11}$ as macromoléculas irão se expandir na turbulência quando a taxa de cisalhamento atingir um valor crítico. No caso do escoamento em um tubo, isto ocorre preferencialmente no seu núcleo, onde se desenvolve o regime turbulento. Na subcamada laminar, próxima da parede do tubo, os novelos poliméricos não se deformam muito. Na região turbulenta, a expansão macromolecular produz um aumento significativo da viscosidade elongacional (definida como a viscosidade ao longo do escoamento). A viscosidade elongacional impede a formação de pequenos vórtices (que são os que dissipam a energia) e reduz o transporte da turbulência no interior do tubo. Em outras palavras, o estiramento da macromolécula ao longo do escoamento, torna muito mais difícil a rotação local do fluido (esta rotação, também conhecida por vorticidade, caracteriza o vórtice). Vários aspectos interessantes são considerados nesta teoria como: o ponto de início da redução de atrito, a correlação com massa molar do polímero, as mudanças no campo de velocidade do escoamento e a máxima redução de atrito que pode ser atingida. No entanto, a teoria não aborda outros aspectos importantes, como as interações polímero-solvente e a flexibilidade da cadeia polimérica.

Ryskin $^{12}$ usou o princípio básico da viscosidade elongacional elaborado por Lumley ${ }^{11}$ para desenvolver uma teoria quantitativa da redução de atrito, baseado no modelo de "iô-iô". Fundamentalmente, o modelo considera a natureza transiente da extensão macromolecular. As macromoléculas se desenrolam quando submetidas ao escoamento extensional, permanecendo a porção central das cadeias alinhadas, enquanto que as partes terminais permanecem enroladas.

As características peculiares da RAH motivaram o Prêmio Nobel de Física, Pierre G. de Gennes, a propor uma abordagem do fenômeno, que foi designada por Teoria Elástica para a redução de atrito hidrodinâmico. De Gennes e Tabor ${ }^{13}$ partiram da teoria geral para a turbulência desenvolvida por Kolmorogov. ${ }^{14}$ Segundo esta teoria, quando um fluido é submetido a um regime de escoamento turbulento, algumas flutuações podem ocorrer no espaço tridimensional do campo de velocidade (ou seja, são criadas vorticidades). Estas flutuações podem variar de tamanho e frequência, e mesmo evoluir. Para entender melhor, pode-se pensar nestas flutuações como um espectro mecânico, na qual existem certas frequências que estão associadas com os diferentes tamanhos dos vórtices. No escoamento, os vórtices grandes podem se transformar em vórtices menores e estes em vórtices ainda menores, gerando todo o espectro mecânico dos mesmos. Os grandes vórtices transportam o fluido sem perda de energia (isto é o que ocorre nas ondas do mar). No entanto, os vórtices muito pequenos, dissipam a energia do escoamento, pois apresentam tamanhos onde os processos de difusão das moléculas do solvente se tornam importantes. Assim, quando o fluido está escoando em um regime turbulento, desenvolve-se um processo na forma de cascata, em que vórtices grandes se transformam em muitos vórtices pequenos. Esta transformação é conhecida por Cascata de Kolmorogov. Fundamentalmente, a Teoria Elástica relaciona a frequência de formação de vórtices com a frequência com que as macromoléculas estiram e contraem no campo de cisalhamento (elas relaxam com um tempo característico, $\lambda_{i}$ ).

O modelo de Rouse-Zimm, ${ }^{15,16}$ que trata da dinâmica de soluções poliméricas diluídas, permite determinar $\lambda_{i}$. De acordo com o modelo, as cadeias poliméricas são representadas por esferas (submoléculas) 
conectadas por molas hookeanas, sendo que o escoamento da solução causa a deformação e o alinhamento da cadeia polimérica no fluxo, consequentemente diminuindo sua entropia. Assim, a energia elástica que é armazenada nas molas é restituída devido ao movimento browniano das esferas, aumentando novamente a entropia do sistema. Zimm ${ }^{16}$ acrescentou ao Modelo de Rouse as interações hidrodinâmicas que uma determinada submolécula causa nas suas vizinhas, ao friccionar com o solvente.

Estes movimentos coordenados das submoléculas produzem um espectro de relaxações macromolecular, correspondentes aos modos coordenados dos estiramentos dos segmentos. O tempo de relaxação de cada modo é dado pela Equação 2:15,16

$$
\lambda_{i}=\frac{M \eta_{s}[\eta]}{0,586 R T \kappa_{i}}
$$

onde $M$ é a massa molar do polímero, $\eta_{s}$ é a viscosidade do solvente, [ ] é a viscosidade intrínseca, $R$ a constante dos gases, $T$ a temperatura na escala absoluta e $\kappa_{i}$ é o autovalor do i-ésimo modo de relaxação coordenado da macromolécula.

Na Figura 3 estão apresentadas representações para alguns dos modos coordenados (para i =1, 2, 3 e 4) para uma cadeia polimérica genérica (adaptadas da ref. 17). Quando menor o valor de i, mais coordenado é o movimento das submoléculas. Assim, o tempo de relaxação é maior quando $i$ é pequeno, e diminui rapidamente quando $i$ aumenta.

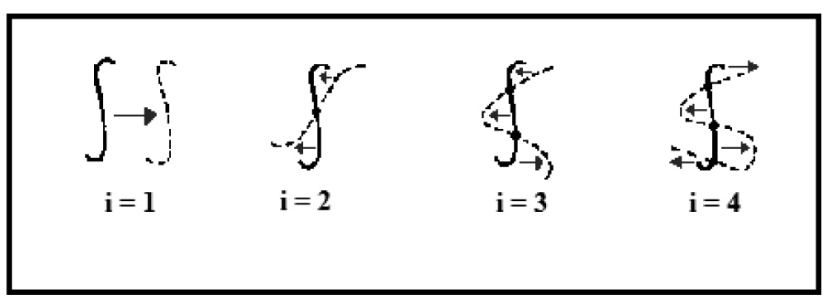

Figura 3. Representações esquemáticas de quatro movimentos coordenados de submoléculas de uma cadeia polimérica. Adaptadas da ref. 17

Considera-se que a maior contribuição para a RAH é aquela onde $i=1$, que está associado com o mais longo tempo de relaxação e que corresponde ao movimento coordenado de todos os segmentos. Neste caso, a Equação 2 pode ser reescrita como: ${ }^{16}$

$$
\lambda_{1}=\frac{M \eta_{s}[\eta]}{0,586 R T}
$$

De Gennes e Tabor consideraram que para as flutuações de velocidades de baixa frequência (correspondentes aos grandes vórtices), muito menores do que $1 / \lambda_{l}$, nenhuma contribuição importante do polímero para o fluxo é importante. Entretanto, há um valor crítico, no qual as duas frequências, a relativa à formação dos vórtices e a relacionada com a relaxação dos polímeros, se igualam e, nesta condição, iniciam os efeitos de redução de atrito. A partir deste ponto, ocorrerá um truncamento da Cascata de Kolmorogov, ou seja, os vórtices, ao atingirem um tamanho (correspondente a uma determinada frequência), serão impedidos de se desenvolverem para vórtices menores, os quais são os responsáveis pela dissipação energética.

Para partículas rígidas (como, por exemplo, um polieletrólito e em baixa força iônica), a teoria Elástica considera que os efeitos de redução de atrito são explicados pela situação limite, na qual o polímero se encontra totalmente estendido ao longo do escoamento e, portanto, algum grau de redução de atrito hidrodinâmico observável é devido apenas à orientação das partículas no escoamento.

\section{AGENTES REDUTORES DE ATRITO HIDRODINÂMICO}

Polímeros, surfatantes, suspensão de partículas insolúveis, tais como grãos finos ou fibras, e polímeros misturados com sabões ou fibras, são alguns tipos de agentes que produzem RAH. ${ }^{9}$ Alguns polímeros naturais, como polissacarídeos, ${ }^{3} \mathrm{DNA}$, colágeno, ${ }^{18} \mathrm{e}$ algumas gomas (como goma guar e xantana) também produzem RAH, porém normalmente requerem maior concentração para se ter o mesmo efeito dos polímeros sintéticos. ${ }^{19}$

Uma característica do fenômeno de RAH em relação ao agente polimérico é a ínfima quantidade de polímero requerida, da ordem de partes por milhão, de polímeros (geralmente lineares) e de elevada massa molar média (da ordem de $\left.10^{6} \mathrm{~g} \mathrm{~mol}^{-1}\right){ }^{3}$ Os polímeros mais estudados em sistemas aquosos são poli(óxido de etileno) (PEO) e poliacrilamida (PAM).$^{5,20-23}$ Índices de até $80 \%$ de redução de atrito podem ser atingidos para estes sistemas em determinadas condições de escoamento. ${ }^{5,21}$ Já para solventes orgânicos, os polímeros mais estudados são poli-isobutileno (PIB) e poliestireno (PS). ${ }^{24-26}$ As estruturas moleculares destes polímeros e de algumas outras substâncias que são capazes de produzir RAH estão apresentadas na Tabela 1.

Outras variáveis como qualidade do solvente e temperatura também afetam a capacidade do polímero de reduzir o atrito hidrodinâmico, uma vez que alteram a conformação macromolecular e, consequentemente, a interação das cadeias poliméricas com a turbulência. A eficiência da redução de atrito é maior em bom solvente do que em mau solvente. ${ }^{26}$

$\mathrm{O}$ fato de o fenômeno ocorrer apenas com polímeros contendo certa massa molar mínima impõe restrições a aplicações de sistemas poliméricos, pois a degradação mecânica causada pelo elevado cisalhamento resulta na diminuição da massa molecular do polímero e, consequentemente, na perda da eficiência da redução de atrito. ${ }^{9}$ Este problema é bastante crítico em sistemas que envolvem recirculação do líquido. Uma classe de agentes redutores de atrito hidrodinâmico muito interessante, que é completamente livre da degradação mecânica e muito eficiente, é baseada em alguns surfatantes. Em determinadas condições, eles podem formar longas micelas, que são designadas por micelas gigantes ou micelas do tipo verme (giant-micelles e worm-like micelles, respectivamente). Tais estruturas são formadas pela mistura, por exemplo, de surfatantes catiônicos e determinados cossolutos. ${ }^{27-29} \mathrm{Um}$ dos sistemas mais estudados envolve a formação de micelas gigantes pela combinação do tensoativo catiônico, brometo de hexadecil trimetil amônio (CTAB) e salicilato de sódio ou potássio, na qual os íons salicilato se interpõem entre as cabeças do surfatante catiônico. Uma representação esquemática da formação destas micelas está apresentada na Figura 4. A especificidade dos cossolutos para a formação das micelas gigantes catiônicas é um tópico ainda não bem estabelecido. Por exemplo, nem todos os isômeros do salicilato produzem a estrutura das micelas gigantes com CTAB.

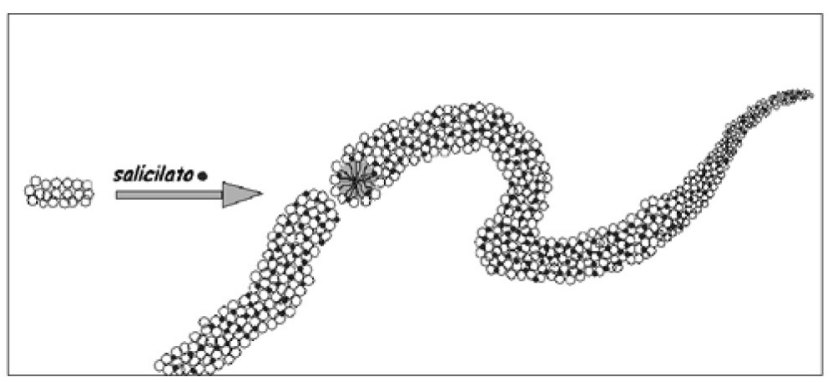

Figura 4. Representação esquemática da formação de micelas gigantes de $C T A B /$ salicilato. As moléculas de salicilato ficam inseridas entre as moléculas de CTAB 
Tabela 1. Fórmulas moleculares de algumas substâncias redutoras de atrito hidrodinâmico

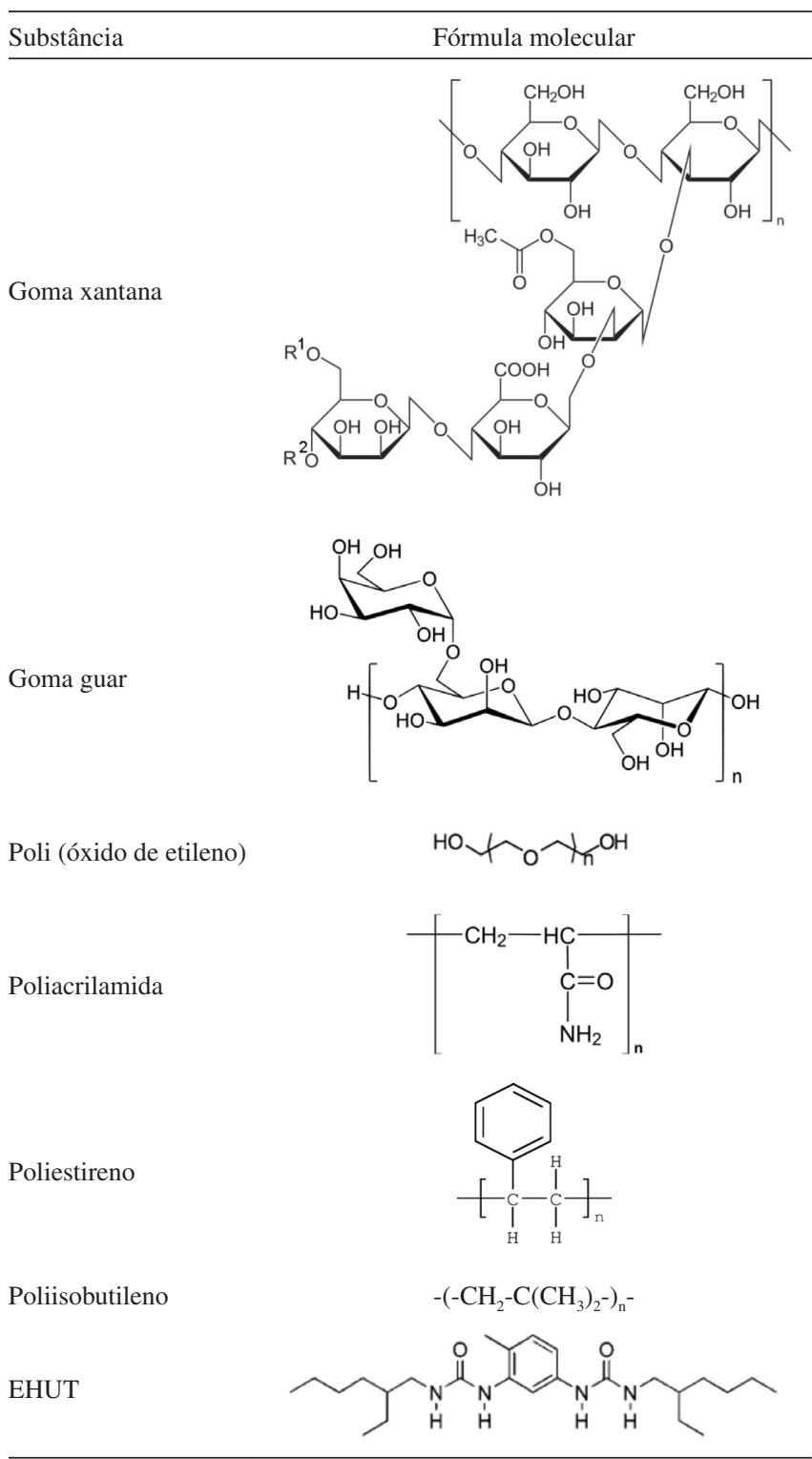

Os longos filamentos formados pela interação das moléculas do CTAB com o salicilato podem ser visualizados por microscopia eletrônica de transmissão obtida em temperaturas criogênicas, como mostrado na Figura 5.

Zakin e colaboradores ${ }^{28,29}$ estudaram uma série de tensoativos comerciais que podem ser utilizados em circuitos de circulação de água sem perda da capacidade de reduzir atrito. As micelas gigantes, ao contrário dos sistemas poliméricos, são sistemas de autoassociação, induzidas por interações não covalentes. Neste caso, são capazes de restaurar a estrutura macromolecular muito rapidamente após alguma cisão (o fenômeno é conhecido por autocura).

$\mathrm{O}$ fato de que apenas longas estruturas moleculares produzem RAH permitiu que fosse determinada a estabilidade térmica de micelas gigantes de CTAB e salicilato, nas mais variadas proporções dos dois componentes. Isto foi feito mantendo-se a solução de micela gigante sob fluxo turbulento com concomitante aumento da temperatura. Observou-se que, em certas temperaturas críticas, as estruturas supramoleculares se rompem, com consequente perda da capacidade de manter a redução do nível de turbulência. ${ }^{30}$

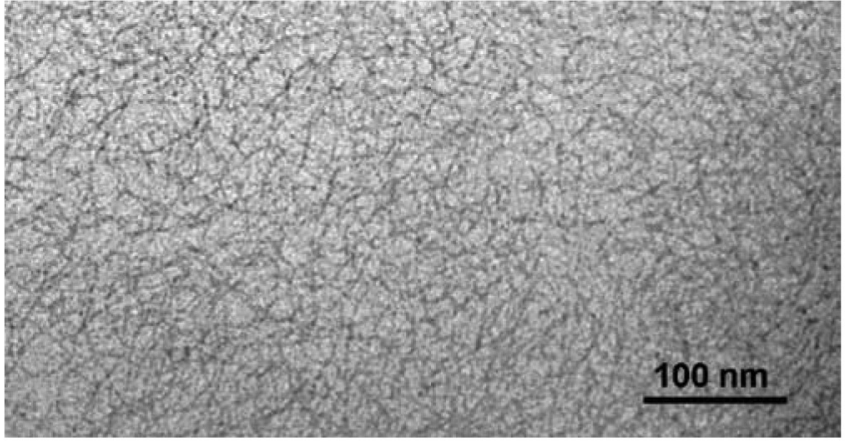

Figura 5. Microscopia eletrônica de transmissão obtida em temperaturas criogênicas de micelas gigantes, aqui vistas como longos filamentos entrelaçados

Recentemente, foi divulgado o primeiro sistema autoarranjado, que espontaneamente forma longas estruturas, capazes de reduzir o atrito hidrodinâmico em hidrocarbonetos. ${ }^{31} \mathrm{~A}$ unidade básica deste polímero não covalente é um derivado de bis-ureia (ver a estrutura molecular na Tabela 1), que se arranja por ligações de hidrogênio, formando estruturas (Figura 6) na forma de longos tubos (com dimensões da ordem $\cong 400 \mathrm{~nm}$, que corresponde a $\mathrm{M}_{\mathrm{n}} \cong 10^{6} \mathrm{~g} \mathrm{~mol}^{-1}$ ) e fitas curtas ( com dimensões $\cong 6 \mathrm{~nm} \mathrm{e}_{\mathrm{n}} \cong 610^{3} \mathrm{~g} \mathrm{~mol}^{-1}$ ). ${ }^{32}$ Os arranjos moleculares na forma de tubo são muito eficientes na redução de atrito em solventes do tipo heptano e tolueno, no entanto, o polímero supramolecular na forma de fita não é capaz de promover RAH, pois não possui o tamanho crítico requerido.
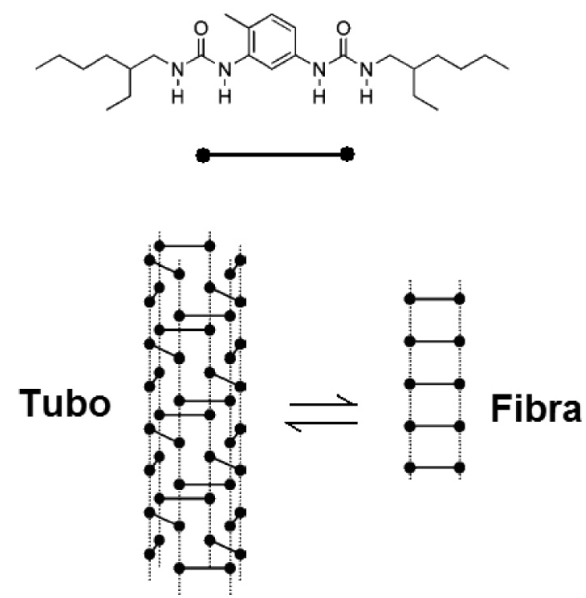

Figura 6. Formação de polímeros supramoleculares de bis-ureia em solventes à base de hidrocarbonetos. Os arranjos moleculares se formam como longos tubos e fitas (mais curtas). Apenas os longos tubos são capazes de reduzir o atrito hidrodinâmico no solvente

\section{TÉCNICAS EXPERIMENTAIS USADAS PARA ESTUDAR O FENÔMENO}

Uma vez que o fenômeno está intrinsecamente ligado a soluções muito diluídas, estudos ao nível molecular, simultâneos à turbulência do escoamento são praticamente impossíveis. Normalmente, parâmetros obtidos em situações quiescentes, como raio de giração, mudança na massa molar, tempo de relaxação do polímero, etc, são usados para correlacionar com a magnitude da redução de atrito medida. Medir a magnitude da redução de atrito hidrodinâmico é relativamente fácil, considerando a magnitude do efeito. Geralmente as medidas de redução de atrito são feitas em tubos capilares, ${ }^{33}$ contendo dois transdutores de pressão colocados em pontos distintos da tubulação (Figura 7A), que medem diferenças de pressão $\left(\mathrm{P}_{1}\right.$ e $\left.\mathrm{P}_{2}\right)$. 

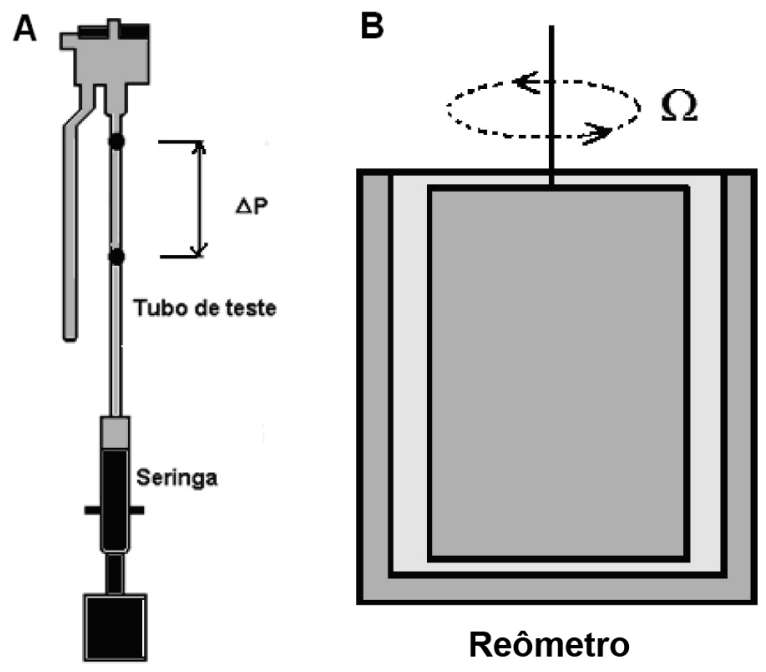

Figura 7. Esquemas de equipamentos usados para medida da redução de atrito hidrodinâmico: A- Reômetro capilar e B-cela de Couette usada em um reômetro rotacional

Uma vez que o agente redutor de atrito hidrodinâmico produz menor perda de carga no fluido, a queda de pressão é menor para a solução $\left(\Delta P_{p}\right)$ do que para o respectivo solvente puro $\left(\Delta P_{s}\right)$. Este tipo de sistema foi usado por Toms em sua descoberta primordial. A porcentagem de redução de atrito hidrodinâmico $(\% R A H)$, para determinado Re, é obtida da Equação 4:

$$
(\% R A H)_{\operatorname{Re}}=\left(\frac{\Delta P_{s}-\Delta P_{p}}{\Delta P_{s}}\right) x 100
$$

Outro sistema que pode ser utilizado é o de discos rotatórios. Neste, a redução de atrito é quantificada em função do torque requerido para manter certa velocidade angular $(\Omega)$ de um disco imerso no fluido.$^{34,35} \mathrm{Na}$ presença do agente redutor de atrito, o torque $\left(T_{p}\right)$ é menor do que para o solvente puro $\left(T_{s}\right)$, e a porcentagem de redução de atrito é definida pela Equação 5:

$$
(\% R A H)_{\Omega}=\left(\frac{T_{s}-T_{p}}{T_{s}}\right) x 100
$$

Recentemente, Nakken e colaboradores ${ }^{36,37}$ apresentaram uma nova técnica para estudar a redução de atrito usando um reômetro contendo sensores cilíndricos concêntricos (Figura 7B). A amostra preenche o compartimento que fica entre os dois cilindros. O princípio desta técnica é muito parecido com o do disco rotatório, mas a elevada sensibilidade do reômetro resulta em medidas de RAH de grande precisão $( \pm 3 \%)$. Um diagrama típico obtido quando se usa esta técnica está apresentado na Figura 8A, na qual se mede o torque aplicado no cilindro rotatório, para mantê-lo rodando em determinada rotação (tipicamente a rotação pode atingir até $1200 \mathrm{rpm}$ ). A técnica foi usada pelos autores para estudar o efeito comparativo da cinética de degradação mecânica de polímeros ${ }^{38}$ e para selecionar os agentes redutores de atrito e suas concentrações ótimas, para amostras de petróleo. ${ }^{39} \mathrm{~A}$ cinética comparativa da degradação mecânica de polímeros sintéticos também foi estudada, usando-se a medida da perda da capacidade dos polímeros em manter a redução de atrito, devido à cisão de suas cadeias (Figura 8B). A grande sensibilidade desta técnica permitiu estudos sobre a estabilidade térmica de micelas gigantes de CTAB e salicilato. ${ }^{30} \mathrm{~A}$ destruição das micelas gigantes devido ao aumento da

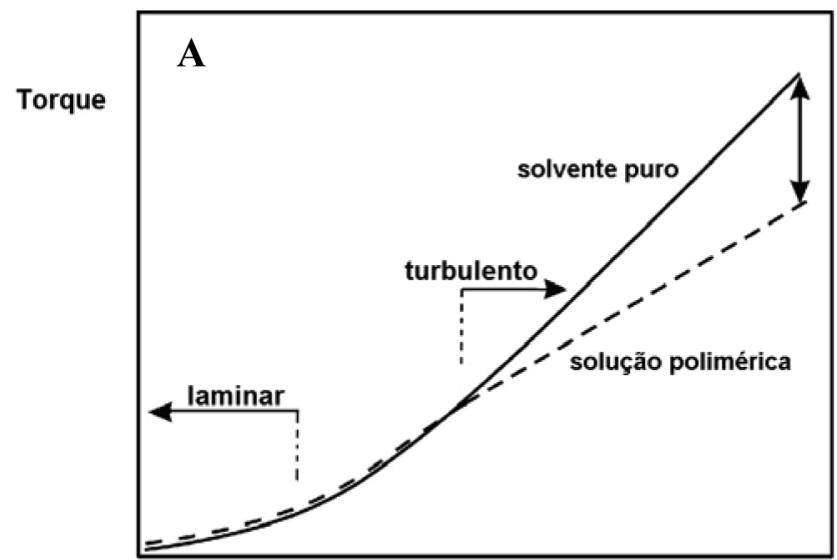

Velocidade Angular
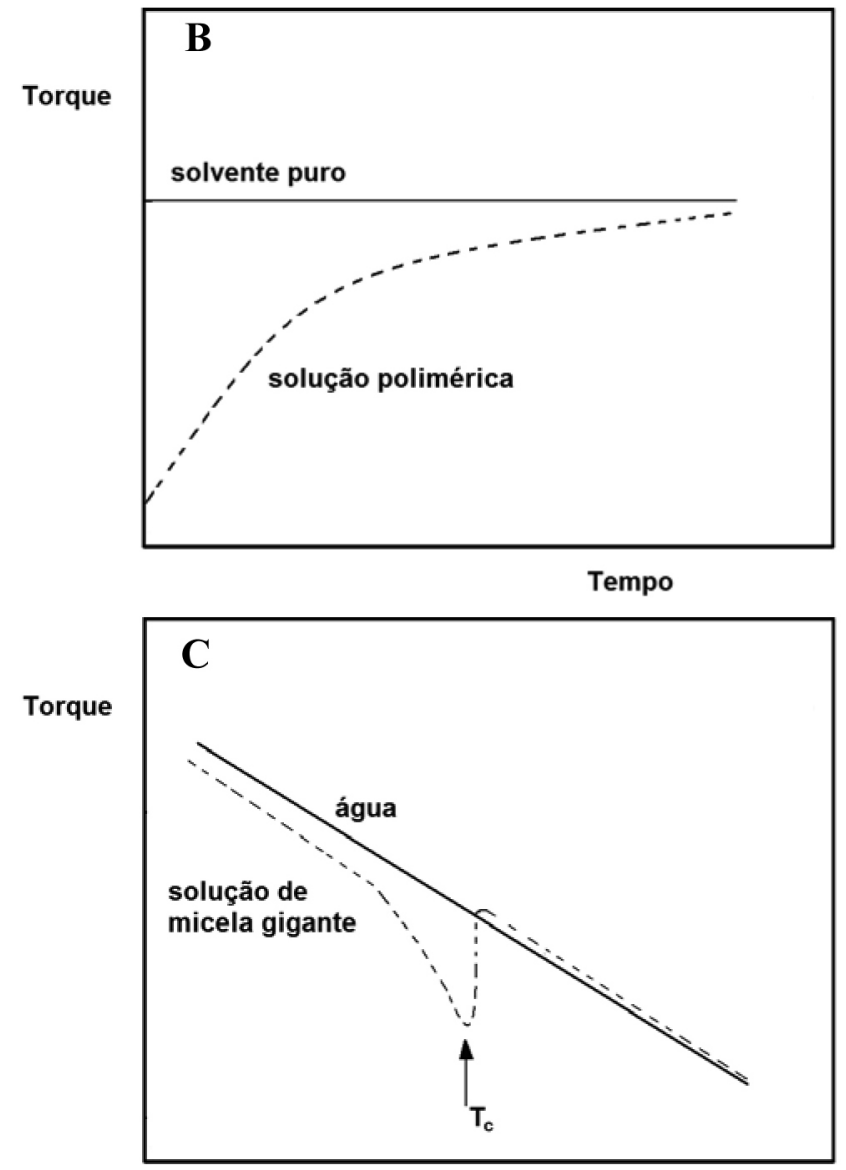

Temperatura

Figura 8. Gráficos genéricos mostrando o comportamento reológico obtido nos estudos usando o sistema descrito na Figura 7B. A: curva de fluxo (torque em função da velocidade angular) para o solvente puro (linha contínua) $e$ para o solvente contendo um polímero redutor de atrito (linha tracejada); B: curva típica em estudos sobre a cinética de degradação mecânica de um polímero, em que a rotação em regime turbulento é mantida fixa; C: curva típica para a estabilidade térmica das micelas gigantes, em que a rotação em regime turbulento é mantida constante

temperatura resulta na imediata perda da capacidade de redução de atrito hidrodinâmico e o nível de turbulência retorna aos valores para a água sem o aditivo. A temperatura de quebra das micelas gigantes é facilmente caracterizada por um pico no diagrama (Figura 8C).

Uma grande inovação em termos de técnica usada para estudar o efeito foi apresentada pelos autores em uma série de artigos 
recentes. ${ }^{40-42} \mathrm{~A}$ técnica se baseia na visualização da dinâmica de deformação do fluido a partir do impacto de uma gota contra uma superfície líquida rasa (splash). Em cerca de 0,02 s após o impacto da gota, forma-se uma estrutura na forma de coroa, que posteriormente colapsa devido à força gravitacional e, principalmente, pela tensão superficial.O colapso da coroa gera uma coluna líquida, conhecida por jato Rayleigh (Figura 9).

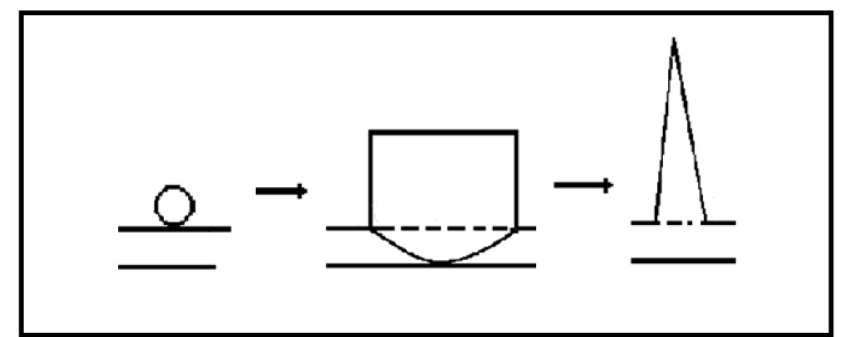

Figura 9. Representação esquemática para três momentos do splash. A figura da esquerda representa o instante do impacto da gota contra a superfície líquida; a do meio, a formação da coroa; a da direita, a formação do jato Rayleigh, que sucede o colapso da coroa

Para os estudos do splash, foi usada uma câmera capaz de filmar em alta velocidade (cerca de 1000 quadros por s). Durante o impacto, a turbulência gerada devido à deformação caótica da massa líquida, e sua interação com o redutor de atrito, resulta em menor dissipação de energia, que pode ser observada nas estruturas formadas no impacto. Nestas estruturas é possível observar a característica elongacional das soluções poliméricas, podendo relacionar o tempo de deformação do fluido com o tempo de relaxação da macromolécula. Uma das estruturas características do splash, o jato Rayleigh, começa a se formar em cerca de $0,04 \mathrm{~s}$ após o impacto da gota contra superfície. Ele mostrou-se muito sensível à presença de agentes redutores de atrito hidrodinâmico. Como apresentado na Figura 10, a menor dissipação da energia do impacto resulta em jatos com alturas muito superiores que as do solvente puro. Um filme comparativo do impacto de uma gota em queda livre (de uma altura de cerca de $1,8 \mathrm{~m}$ ) sobre a superfície da água pura e de uma solução de PEO pode ser visto em: http://www.youtube.com/ watch?v=VudmLmjfgLQ (para o splash em água pura) e em http:// www.youtube.com/watch?v=XKnu-ZJe0L8\&NR=1 (para o splash em água contendo o redutor de atrito hidrodinâmico).
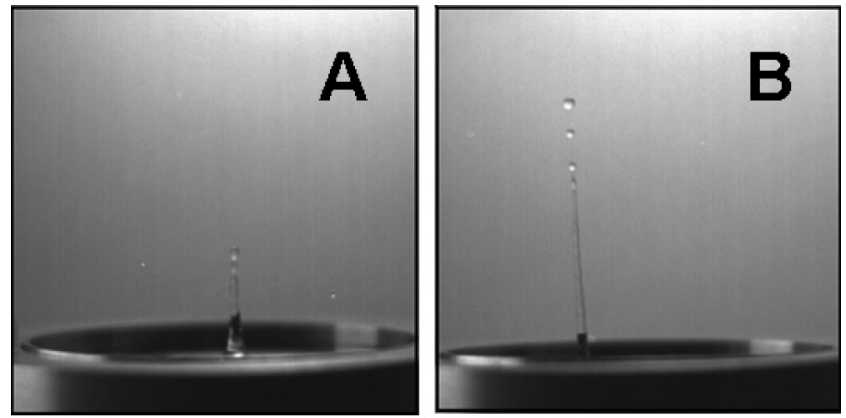

Figura 10. Duas fotografias comparativas para o jato Rayleigh, produzidas por gotas que caíram livremente de uma altura de cerca de 1,8 $\mathrm{m}$, contra uma superfície rasa. Em A, o splash envolveu apenas água e em B, uma solução de PEO

\section{APLICAÇÕES}

Em princípio, o Efeito Toms pode ser aplicado para qualquer fluido que escoe em regime turbulento, visando assim facilitar o deslizamento do fluido, com menor dispêndio de energia. Algumas aplicações envolvem hidrotransporte de sólidos, como minérios, e também de partículas sólidas, como areia, nos processos de fratura de poços de petróleo, em sistema de circulação de água quente (como em distritos de países de clima frio), em sistemas hidráulicos e mesmo em estudos preliminares para o melhoramento do fluxo sanguíneo em artérias parcialmente bloqueadas. ${ }^{19} \mathrm{O}$ fenômeno também tem potencial para uso em operações de combate a incêndio para bombeamento de água ${ }^{5}$ (Figura 1) e de espumas especiais para extinção de incêndios em combustíveis. ${ }^{43}$ Uma das aplicações mais significativas da redução de atrito hidrodinâmico está relacionada com o bombeamento de petróleo ao longo do oleoduto que corta o Alasca, ligando as Baias de Prudhoe a Valdez, por uma extensão de $1287 \mathrm{~km}$. Cerca de $30 \%$ de redução de atrito é atingida; porém, a cada $100 \mathrm{~km}$ se faz necessária a reaplicação do redutor de atrito devido à perda de eficácia ocorrida pela degradação das macromoléculas. ${ }^{1}$ Assim, uma boa redução de energia elétrica pode ser obtida usando-se bombas operando com menor potência para manter a mesma vazão ou, alternativamente, maior vazão pode ser obtida com as mesmas bombas operando na potencia nominal. A Petrobras utiliza esta estratégia em operações de manutenção de dutos, em que a adição de agentes redutores de atrito em determinado duto compensa, em parte, a diminuição da vazão total, devido ao fechamento do duto em manutenção. O Brasil é o principal produtor mundial de biocombustíveis, principalmente etanol. $\mathrm{O}$ vasto território nacional e a necessidade de bombeio por longos álcooldutos nos mobilizou a desenvolver agentes redutores de atrito específicos para este combustível. ${ }^{44}$

Na natureza é bem conhecido que o deslizamento de golfinhos é facilitado pela secreção de um biopolímero capaz de reduzir o atrito do seu corpo com a água do mar. Mimetizando este animal, o efeito já foi usado para melhorar o deslizamento de navios, submarinos e mesmo torpedos. Nestes casos, uma tinta contendo o agente redutor de atrito é usada para recobrir a estrutura externa do dispositivo. ${ }^{19}$ Recentemente foi demonstrado que pequena redução de atrito pode ser obtida pela geração de microbolhas na proa de navios. ${ }^{45}$

Em uma época onde a economia de energia se mostra essencial, estratégias que facilitem o transporte de líquidos por longas distâncias ou reduzam sua fricção com superfícies sólidas são extremamente bem vindas.

\section{AGRADECIMENTOS}

À FAPESP, ao CNPq e PRP-UNICAMP pelo apoio financeiro. À editora Elsevier por permitir a reprodução da fotografia apresentada na Figura 1.

\section{REFERÊNCIAS}

1. Kulicke, W.-M.; Andreis, M.; Gräger, H.; Koening, J. L.; Köter, M.; Advances in Polymer Science, Polymer Characterization/Polymer Solutions, Springer-Verlag: Berlim, 1989, vol. 89.

2. White, A.; Hemmings, J. A. G. Em Drag reduction by additives: review and bibliography; White, A.; Hemmings, J. A. G., eds.; BHRA Fluid Engineering: Bedford, 1976.

3. Shenoy, A. V.; Colloid Polym. Sci. 1984, 262, 319.

4. Leuchtag, H. R.; Phys. Today 1978, 31, 17.

5. Bailey, F. E.; Koleske, J. V.; Poly(ethylene oxide), Academic Press Inc.: New York, 1976.

6. Veja, por exemplo, em: http://en.wikipedia.org/wiki/Turbulence, acessada em Outubro 2010.

7. Screenivasan, K.; White, C. M.; J. Fluid Mech. 2000, 409, 149. 
8. Goodwin, J. W.; Hughes, R. W.; Rheology for chemists-An introduction, The Royal Society of Chemistry: Cambridge, 2000.

9. Morgan, S. E.; McCormick, C. L.; Prog. Polym. Sci. 1990, 15, 507.

10. Virk, P. S.; Merrill, E. W.; Smith, K. A.; J. Fluid Mech. 1967, 30, 305.

11. Lumley, J. L.; Ann. Rev. Fluid Mech. 1969, 1, 367.

12. Ryskin, G.; Phys. Rev. Lett. 1987, 59, 2059.

13. De Gennes, P. G.; Introduction to Polymer Dynamics, Cambridge University Press: New York, 1990.

14. A teoria pode ser encontrada em: Landahl, M. T.; Mollo-Christensen, E.; Turbulence and Random Processes in Fluid Mechanics, Cambridge University Press: New York, $2^{\text {nd }}$ ed., 1992.

15. Rouse Jr., P. E.; J. Chem. Phys. 1953, 21, 1272.

16. Zimm, B. H.; J. Chem. Phys. 1956, 24, 269.

17. Hershey, H. C.; Zakin, J. L.; Chem. Eng. Sci. 1967, 22, 1847.

18. Berman, N. S.; Ann. Rev. Fluid Mech. 1978, 10, 27.

19. Sellin, R. H. J.; Hoyt, J. W.; Scrivener, O.; J. Hydraul. Res. 1982, $20,29$.

20. Hoyt, J. W.; J. Polym. Sci, Polym. Lett. 1966, 4, 713.

21. Peyser, P.; Little, R. C.; J. Appl. Polym. Sci. 1971, 15, 2623.

22. Cowan, M. E.; Garner, C.; Hester, R. D.; McCormick, C. L.; J. Appl. Polym. Sci. 2001, 82, 1222.

23. Kenis, P. R.; J. Appl. Polym. Sci. 1971, 15, 607.

24. Ram, A.; Finkelstein, E.; Elata, C.; I \& EC Process Design and Development 1967, 6, 309.

25. Choi, H. J.; Kim, C. A.; Jhon, M. S.; Polymer 1999, 40, 4527.

26. Kim, C. A.; Choi, H. J.; Sung, J. H.; Lee, H. M.; Jhon, M. S.; Macromol. Symp. 2005, 222, 169.

27. Cooper-White, J. J.; Crooks, R. C.; Boger, D. V.; Colloids Surf., A 2002, 210,105 .
28. Zhang, Y.; Schmidt, J.; Talmon, Y.; Zakin, J. L.; J. Colloid Interface Sci. 2005, 286, 696.

29. Qi, Y. Y.; Zakin, J. L.; Ind. Eng. Chem. Res. 2002, 41, 6326.

30. Rodrigues, R. K; da Silva, M. A.; Sabadini, E.; Langmuir 2008, 24, 13875.

31. Sabadini, E.; Francisco, K. R.; Bouteiller, L.; Langmuir 2010, 26, 1482.

32. Bellot, M.; Bouteiller, L.; Langmuir 2008, 24, 14176.

33. Little, R. C.; J. Appl. Polym. Sci. 1971, 15, 3117; Kulik, V. M.; Exp. Fluids 2001, 31, 558

34. Kalashnikov, V. N.; J. Non-Newtonian Fluid Mech. 1998, 75, 209; Koeltzsch, K

35. Qi, Y.; Brodkey, R. S.; Zakin, J. L.; Exp. Fluids 2003, 34, 515.

36. Nakken, T.; Tande, M.; Elgsaeter, A.; J. Non-Newtonian Fluid Mech. 2001, 97, 1

37. Nakken, T.; Tande, M.; Nyström, B.; Eur. Polym. J. 2004, 40, 181

38. Bizotto, V. C.; Sabadini, E.; J. Appl. Polym. Sci. 2008, 110, 1844.

39. Sabadini, E.; da Silva, M. A.; Carvalho, C. H.; Rocha, N. O.; Energy Fuels 2009, 23, 4529.

40. Sabadini, E.; Alkschbirs, M. I.; Exp. Fluids 2002, 33, 242.

41. Sabadini, E.; Alkschbirs, M. I.; Bizotto, V. C.; de Oliveira, M.; Langmuir 2004, 20, 11315.

42. Sabadini, E.; Alkschbirs, M. I.; J. Phys. Chem. B 2004, 108, 1183.

43. Sabadini, E.; Figueredo, R.; Colloids Surf., A 2003, 215, 77.

44. Sabadini, E.; Francisco, K. R.; Pedido de patente protocolado sob número: 018090002768.

45. Kitagawa, A.; Hishida, K.; Kodama, Y.; Exp. Fluids 2005, 38, 466. 